\title{
Urinary Tract Infection in Hemodialysis-Dependent End-Stage Renal Disease Patients
}

\author{
Kaori Yamashita $\mathbb{D}^{1,2}$, Yudai Ishiyama', Maki Yoshino', Hidekazu Tachibana', Daisuke Toki', \\ Ryuichiro Konda ${ }^{2}$, Tunenori Kondo' \\ 'Department of Urology, Tokyo Women's Medical University Medical Center East, Tokyo, Japan; ${ }^{2}$ Tomioka Clinic, Tokiwa Foundation, Fukushima, \\ Japan
}

Correspondence: Kaori Yamashita, Department of Urology, Tokyo Women's Medical University Medical Center East, 2-I-I0, Nishi-ogu, Arakawa-ku, Tokyo, I I6-8567, Japan, Tel +8I 338I0 I I I , Fax +8I 338940282, Email kmurology@yahoo.co.jp

\begin{abstract}
Introduction: The aim of this study was to analyze urinalysis findings and urinary bacterial culture in hemodialysis-dependent endstage renal disease patients. The research goal was to understand the proportion, risk factors, and the causative organisms of urinary tract infection in hemodialysis-dependent end-stage renal disease patients.

Materials and Methods: Between May 2020 and June 2021, this study included 100 hemodialysis-dependent end-stage renal disease patients (50 male patients and 50 female patients). The urine underwent microscopic examination, pyuria was defined as $\geq 5$ white blood cells per high-power field, and urinary bacterial cultures were conducted for patients with pyuria. Bacteriuria was defined as $\geq 10^{4}$ colony-forming units $/ \mathrm{mL}$ in men and $\geq 10^{5}$ colony-forming units $/ \mathrm{mL}$ in women. Daily urine output was investigated by oral listening. Postvoiding residual urine volume was measured.

Results: Fifty-six percent of male patients and 30\% of female patients had normosthenuria, $24 \%$ of male patients and $38 \%$ of female patients had pyuria, and $20 \%$ of male patients and $32 \%$ of female patients had a urinary tract infection. A comparison of normosthenuria and urinary tract infection revealed no statistically significant difference in age, time on dialysis, daily urine output, and postvoiding residual urine volume. The proportion of female patients among those with normosthenuria was $34.8 \%$, whereas the proportion of female patients among those with UTI was $61.5 \%$. Urinary bacterial cultures showed that the major causative organisms were Escherichia coli (45\%; 18/40 cultures) and extended spectrum beta-lactamase-producing Escherichia coli (17.5\%; $7 / 40$ cultures).

Conclusion: The incidence of urinary tract infection was higher in female patients than in male patients. The proportion of resistant bacteria as the causative organisms was high in hemodialysis-dependent end-stage renal disease patients. Urinary bacterial culture should be checked while patients are able to void urine.
\end{abstract}

Keywords: bacteriuria, pyuria, renal dialysis, urinary tract infection, urine

\section{Introduction}

Urinary tract infection (UTI) is the second-ranked infectious disease globally and is a global concern, based on the economic and public health points of view. ${ }^{1}$ In recent years, the number of UTIs caused by multidrug-resistant microbial strains has significantly risen, and multidrug-resistant microbial strains have been a clinical concern in public healthcare systems. $^{2}$

UTIs affect male and female individuals, adults, pregnant women, children, young adults, and infants, ${ }^{1}$ and are common in patients with hemodialysis-dependent end-stage renal disease (ESRD). ${ }^{3-5}$ However, taking a urine test is difficult for hemodialysis-dependent ESRD patients. In many hemodialysis clinics and hospitals, routine examinations do not include urinalysis and urinary bacterial cultures, and hemodialysis-dependent ESRD patients may be hesitant to complain to physicians about urination symptoms during physicians' round because of the lack of privacy. 
With regard to UTIs, the symptoms of bacterial cystitis (eg, pollakisuria, urgency, and sense of residual urine or miction pain) affect the quality of life, and pyelonephritis might become urosepsis, ${ }^{6,7}$ which is a life-threatening disease, especially in hemodialysis-dependent ESRD patients. Understanding urinalysis and urinary bacterial cultures in hemodialysis-dependent ESRD patients is necessary.

As far as we know, some reports have been published about the features of urinalysis and urinary bacterial cultures in hemodialysis-dependent ESRD patients. However, the features of urinalysis and urinary bacterial culture, and the proportion of drug-resistant bacteria such as extended spectrum beta-lactamase (ESBL)-producing strains in these patients have not been clarified. We report in this paper the outcomes of urinalysis, urinary bacterial cultures, the proportion of ESBL-producing strains and patients' urination background in dialysis-dependent ESRD patients.

\section{Materials and Methods}

This prospective study was conducted at Tokyo Women's Medical University Center East (Tokyo, Japan) and at Tomioka Clinic (Iwaki City, Fukushima Prefecture, Japan). It was approved by the Institutional Review Board of Tokyo Women's Medical University (registration no. 5583) and conducted in accordance with the Helsinki Declaration. Informed consent was obtained from all included patients.

Between May 2020 and June 2021, we recruited hemodialysis-dependent ESRD patients who voided urine at least once daily and who had been on hemodialysis for more than 1 month. We included patients of all ages, patients who had ESRD from any cause, and patients with certain risk factors for UTIs such as diabetes or benign prostate hyperplasia. However, patients were excluded if they had miction pain or fever, had undergone antibiotic treatment in the previous 1 week, had undergone bladder catheterization, had a urinary stone or urological abnormality, were pregnant, or used steroidal drugs.

Urine was sampled once for each patient. Clean-catch midstream urine was obtained before or after hemodialysis and processed within 2 hours after voiding. The voided urine was centrifuged. The supernatant was poured out, and the sediment was transferred to a clean slide, and thereafter underwent microscopic examination using the standard method at a laboratory in Tokyo Women's Medical University Center East or at Tomioka Clinic. Pyuria was defined as $\geq 5$ white blood cells per high-power field. Urinary bacterial cultures were conducted for patients with pyuria. Urinary bacterial culture analyses were conducted by using the WalkAway 96 plus System (Beckman Coulter, Brea, CA, USA) at the laboratory in Tokyo Women's Medical University Center East or by using the MALDI Biotyper System (Bruker, Billerica, MA, USA) at Kotobiken Medical Laboratories, Inc., Central Laboratory Tohoku. Bacteriuria was defined as $\geq 10^{4}$ colony-forming units (CFU) $/ \mathrm{mL}$ in male patients and $\geq 10^{5} \mathrm{CFU} / \mathrm{mL}$ in female patients. ${ }^{8}$ Antimicrobial susceptibility testing was conducted by using the WalkAway 96 plus System at the laboratory in Tokyo Women's Medical University Center East or by using Dry Plate Eiken at Kotobiken Medical Laboratories, Inc., Central Laboratory Tohoku. Escherichia coli, Klebsiella pneumoniae, Proteus mirabilis were detected as the causative organism, ESBL-producing strain detection was tested by using the MASTDISCS Combi AmpC \& ESBL ID Set (Mast Group, Ltd., Bootle, United Kingdom) at the laboratory in Tokyo Women's Medical University Center East or by using the disc diffusion method at Kotobiken Medical Laboratories, Inc., Central Laboratory Tohoku. The susceptibility of strains to the following 16 antimicrobial agents was tested: ampicillin, cefazolin, cefaclor, cefotaxime, ceftazidime, cefmetazole, imipenem/cilastatin, meropenem, ampicillin/sulbactam, tazobactam/piperacillin, amikacin, gentamicin, minocycline, levofloxacin, ciprofloxacin, and fosfomycin.

Daily urine output was investigated by using information in a patient's bladder diary or the medical record. The lack of miction pain and fever was investigated by oral listening. Postvoiding residual urine volume was measured by using the BladderScan BVI6100 bladder volume device (Verathon Inc, Bothell, WA, USA). Statistical analysis was conducted by using JMP Pro 16 (SAS Institute, Tokyo, Japan). Qualitative parameters were compared by using the $\chi^{2}$ test. Quantitative parameters were compared by using the unpaired two-sample $t$-test. $P$-values less than 0.05 were significant.

\section{Results}

This study included 100 hemodialysis-dependent ESRD patients (50 men and 50 women). The cause of ESRD were diabetic nephropathy (37 patients: 17 male patients and 20 female patients); nephrosclerosis (19 patients: 11 male 
Table I Sex Differences in the Background Characteristics of Hemodialysis-Dependent ESRD Patients

\begin{tabular}{|l|l|l|l|}
\hline & Male Patients & Female Patients & P-value \\
\hline $\begin{array}{l}\text { No. of patients } \\
\text { Age (years) }\end{array}$ & 50 & 50 & \\
$\quad$ Mean \pm SD & $69.9 \pm 12.8$ & $68.5 \pm 14.4$ & 0.58 \\
$\quad$ Median (range) & $71(34-91)$ & $71(37-92)$ & \\
Time on dialysis treatment (months) & $16.0 \pm 21.0$ & $27.4 \pm 28.0$ & 0.06 \\
$\quad$ Mean \pm SD & $8.5(1-84)$ & $23(1-132)$ & \\
Median (range) & $17(34 \%)$ & $20(40 \%)$ & \\
Cause of CKD, n (\%) & $11(22 \%)$ & $8(16 \%)$ & \\
Diabetes & $4(8 \%)$ & 0 & \\
Nephrosclerosis & $2(4 \%)$ & $21(22 \%)$ & \\
Chronic glomerulonephritis & $16(32 \%)$ & $2 \%)$ & \\
IgA nephropathy & & \\
Other & & & \\
\hline
\end{tabular}

Abbreviations: ESRD, end-stage renal disease; SD, standard deviation; CKD, chronic kidney disease; lgA, immunoglobulin A.

patients and 8 female patients); chronic glomerulonephritis (4 male patients); IgA nephropathy (3 patients: 2 male patients and 1 female patient); and other (37 patients: 16 male patients and 21 female patients). The mean age of the male patients and female patients was 69.9 years and 68.5 years, respectively. The mean time on dialysis treatment of the male patients and female patients was 16.0 months and 27.4 months, respectively. A comparison of male and female patients revealed no statistically significant difference with regard to the cause of ESRD, age, and time on dialysis treatment. Table 1 shows the background characteristics of the hemodialysis-dependent ESRD patients.

Forty-three patients (28 male patients and 15 female patients) had normosthenuria (ie, nonpyuria and nonbacteriuria), 31 patients (12 male patients and 19 female patients) had pyuria only, 0 patients had bacteriuria only, and 26 patients (10 male patients and 16 female patients) had both pyuria and bacteriuria; these 26 patients were diagnosed as having a UTI. As a result, $43 \%$ of all patients, $56 \%$ of male patients, and $30 \%$ of female patients had normosthenuria; $31 \%$ of all patients, $24 \%$ of male patients, and $38 \%$ of female patients had pyuria; and $26 \%$ of all patients, $20 \%$ of male patients, and $32 \%$ of female patients had a UTI (Figure 1). All 26 patients who had UTI did not have miction pain.

The mean age of patients with normosthenuria and UTI was 67.1 years and 70.1 years, respectively. The mean time on dialysis treatment of patients with normosthenuria and UTI was 17.7 months and 24.5 months, respectively. The mean daily urine output of patients with normosthenuria and UTI was $548 \mathrm{~mL}$ and $421.5 \mathrm{~mL}$, respectively. The postvoiding

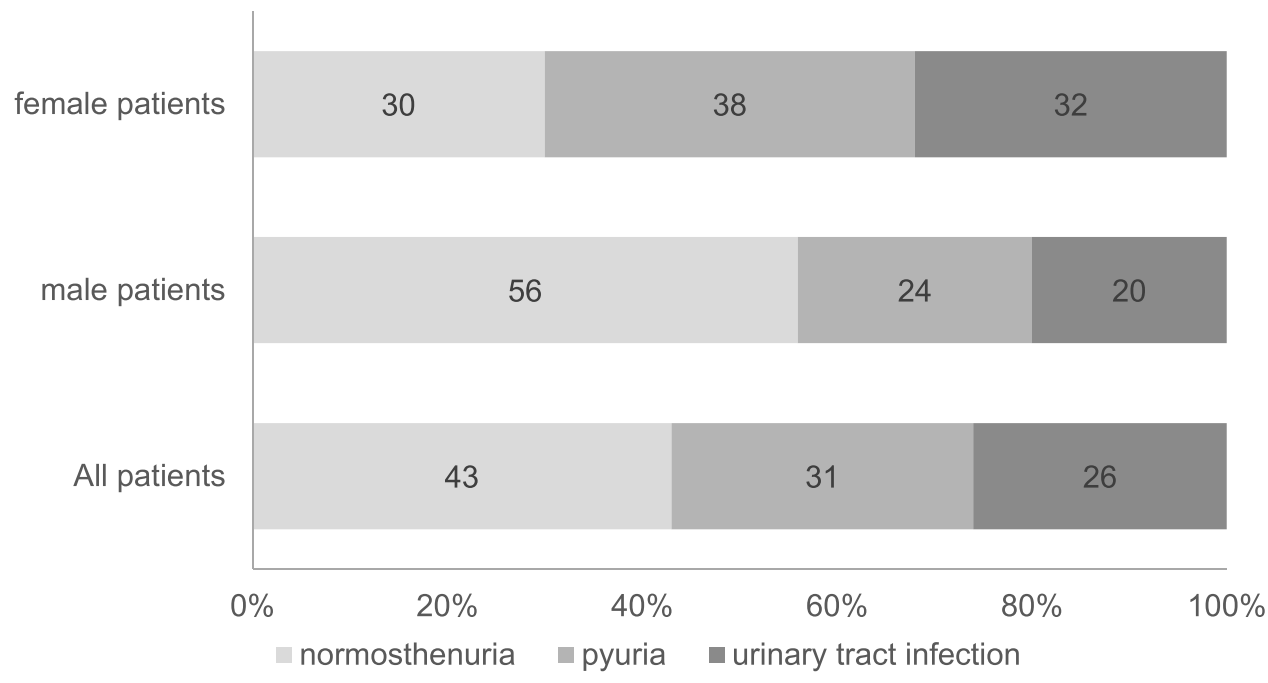

Figure I The proportion of normosthenuria, pyuria, and urinary tract infection in hemodialysis-dependent end-stage renal disease patients. 
Table 2 A Comparison of Urinary Findings Between Normosthenuria and UTI in Hemodialysis-Dependent ESRD Patients

\begin{tabular}{|c|c|c|c|}
\hline & Normosthenuria & UTI & $P$-value \\
\hline No. of patients & 43 & 26 & \\
\hline Age (years) & & & 0.36 \\
\hline Mean \pm SD & $67.1 \pm 15.4$ & $70.1 \pm 10.9$ & \\
\hline Median (range) & $69(34-91)$ & $70(26-92)$ & \\
\hline Time on dialysis treatment (months) & & & 0.23 \\
\hline Mean \pm SD & $17.7 \pm 19.7$ & $24.5 \pm 27.4$ & \\
\hline Median (range) & $8(I-70)$ & $20.5(I-84)$ & \\
\hline Daily urine output (mL) & & & 0.32 \\
\hline Mean \pm SD & $548 \pm 577$ & $421.5 \pm 433.8$ & \\
\hline Median (range) & $300(30-2300)$ & $290(20-1500)$ & \\
\hline Postvoiding residual volume $(\mathrm{mL})$ & & & 0.92 \\
\hline Mean \pm SD & $13.1 \pm 16.2$ & $15.4 \pm 18.8$ & \\
\hline Median (range) & $0(0-4 I)$ & $0(0-50)$ & \\
\hline Presence of diabetic patients, $\mathrm{n}(\%)$ & $17(39.5 \%)$ & 7 (26.9\%) & 0.31 \\
\hline Presence of female patients, $\mathrm{n}(\%)$ & 15 (34.8\%) & $16(61.5 \%)$ & 0.04 \\
\hline
\end{tabular}

Abbreviations: ESRD, end-stage renal disease; UTI, urinary tract infection; SD, standard deviation.

residual volume of patients with normosthenuria and UTI was $13.1 \mathrm{~mL}$ and $15.4 \mathrm{~mL}$, respectively. The proportion of diabetic patients with normosthenuria and UTI was $39.5 \%$ and $26.9 \%$, respectively. A comparison of normosthenuria and UTI in hemodialysis-dependent ESRD patients revealed no statistically significant difference in age, time on dialysis treatment, daily urine output, postvoiding residual urine volume and the proportion of diabetic patients. The proportion of female patients among those with normosthenuria was $34.8 \%$, whereas the proportion of female patients among those with UTI was $61.5 \%$. The proportion of female patients was statistically higher among patients with UTI than among patients with normosthenuria (Table 2).

Among a cumulative total of 40 urinary bacterial cultures from the 26 hemodialysis-dependent ESRD patients who had UTI, 75\% (30/40) of cultures had gram-negative bacteria and 25\% (10/40) of cultures had gram-positive bacteria. The major causative organisms detected were Escherichia coli (45\%; 18/40 cultures) and ESBL-producing Escherichia coli $(17.5 \%$; 7/40 cultures) (Table 3). The susceptible rate of seven ESBL-producing Escherichia coli strains was $100 \%$ for cefmetazole, imipenem/cilastatin, meropenem, tazobactam/piperacillin, and amikacin. However, these strains were totally resistant to ampicillin, cefazolin, cefaclor, cefotaxime, and ceftazidime (Figure 2).

Table 3 Sex Differences in the Bacterial Cultures of Hemodialysis-Dependent ESRD Patients with Bacteriuria

\begin{tabular}{|l|l|l|l|}
\hline & Male Patients (n) & Female Patients (n) & Total n (\%) \\
\hline Gram-negative & II & 19 & $30(75)$ \\
Escherichia coli & 5 & 13 & $18(45)$ \\
ESBL-producing Escherichia coli & 2 & 5 & $7(17.5)$ \\
Klebsiella pneumoniae & $\mathrm{I}$ & 4 & $5(12.5)$ \\
Acinetobacter baumannii & 2 & 0 & $2(5)$ \\
Proteus mirabilis & $\mathrm{I}$ & 0 & $\mathrm{I}(2.5)$ \\
Enterobacter cloacae & 0 & $\mathrm{I}$ & $\mathrm{I}(2.5)$ \\
Pseudomonas aeruginosa & $\mathrm{I}$ & $\mathrm{I}$ & $2(5)$ \\
Citrobacter braakii & $\mathrm{I}$ & 0 & $\mathrm{I}(2.5)$ \\
Gram-positive & 3 & 7 & $10(25)$ \\
Enterococcus faecium & 0 & 5 & $5(12.5)$ \\
Group B streptococcus & 2 & 2 & $4(10)$ \\
Coagulase-negative staphylococci & $\mathrm{I}$ & 0 & $\mathrm{I}(2.5)$ \\
\hline
\end{tabular}

Abbreviations: ESRD, end-stage renal disease; ESBL, extended spectrum beta-lactamase. 


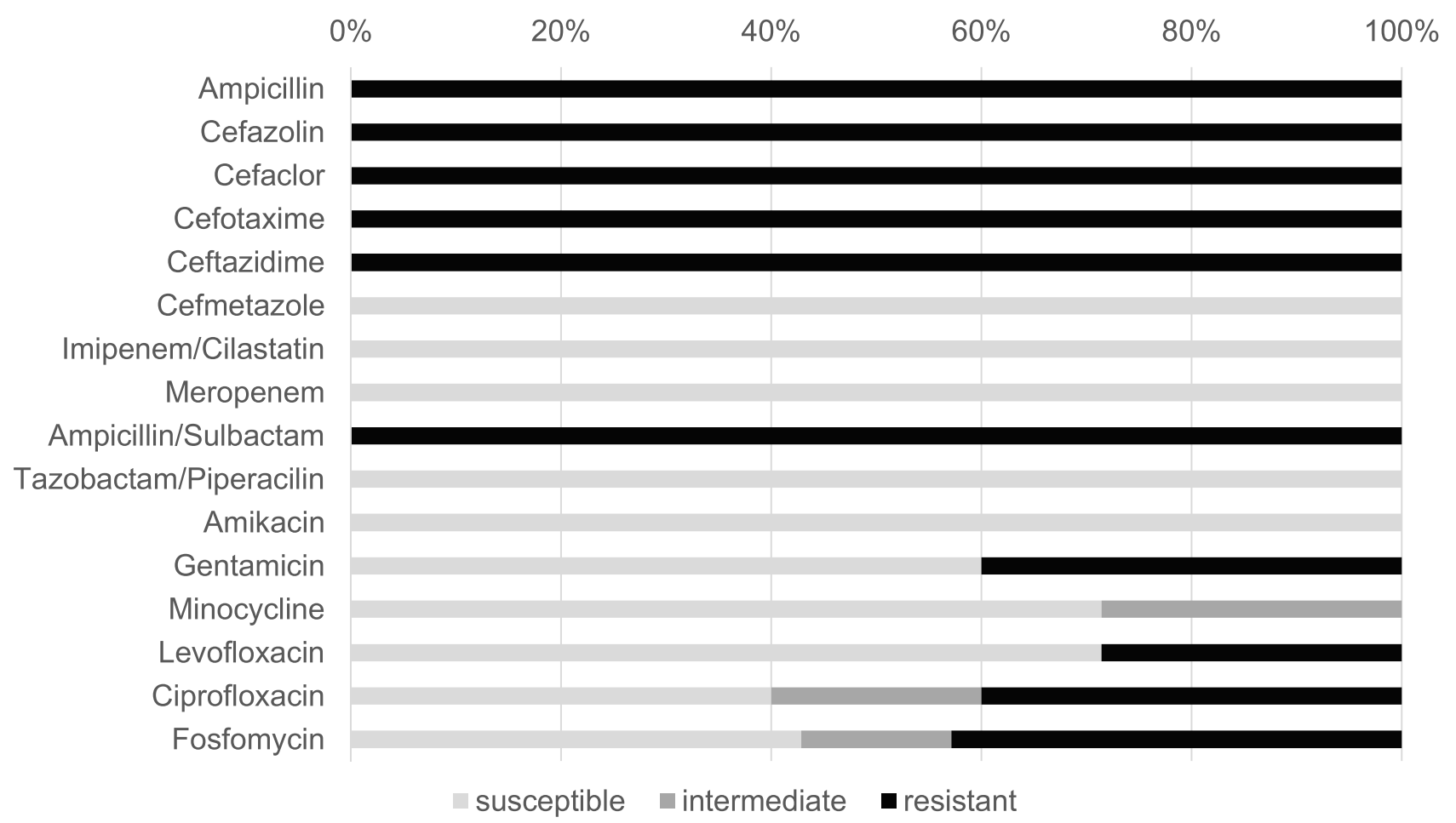

Figure 2 The antimicrobial susceptibility of extended spectrum beta-lactamase-producing Escherichia coli was categorized as susceptible, intermediate, or resistant. Sixteen antibacterial drugs were tested with seven strains of extended spectrum beta-lactamase-producing Escherichia coli.

\section{Discussion}

UTIs are common in chronic kidney disease patients before starting maintenance dialysis therapy ${ }^{9-11}$ and in hemodialysis-dependent ESRD patients. ${ }^{3-5}$ Our study showed that $26 \%$ of hemodialysis-dependent ESRD patients $(20 \%$ male patients and $32 \%$ female patients) were diagnosed as having a UTI. The proportion of UTI among hemodialysisdependent ESRD patients has been reported as $22.5 \%-33 \% .^{3-5}$ The result of our study was compatible with those of past research. ${ }^{3-5}$ In our study, the proportion of UTI in female patients was 1.6 times that of male patients. The high prevalence of UTI in female patients may be associated with the female anatomy. ${ }^{1}$

Bacterial cystitis is one type of UTI. The symptoms of bacterial cystitis are pollakisuria, urgency, sense of residual urine and miction pain. Proof of the existence of these symptoms, pyuria, and bacteriuria are required to diagnose a patient as having bacterial cystitis. We found that hemodialysis-dependent ESRD patients had pyuria and bacteriuria, even though they did not complain about miction pain. We recommend that urinalysis and bacterial cultures should be investigated in advance while hemodialysis-dependent ESRD patients are able to void urine, although they do not complain about miction pain.

A controversial issue is whether pyuria is a useful marker of UTI in hemodialysis-dependent ESRD patients. Manhal et $\mathrm{al}^{3}$ reported that the sensitivity and specificity of pyuria screening for UTI were $60 \%$ and $80 \%$, respectively, and that pyuria was a good marker for significant bacteriuria. However, the positive predictive value of pyuria screening for UTIs was $35.5 \%-45 \%$; therefore, pyuria was not a good marker for UTIs detection. Investigators have concluded that samples positive for pyuria should be cultured to confirm a UTI. ${ }^{12,13}$ Oikonomou and Alhaddad ${ }^{14}$ reported that there was no association between pyuria and fever, sepsis in hemodialysis-dependent ESRD patients, urinalysis was not a reliable diagnostic tool in febrile and/or septic hemodialysis-dependent ESRD patients, and a urine culture was needed. Our study revealed that the positive predictive value of pyuria screening for UTI was $45.6 \%$ (26/57). Thus, we concluded that pyuria was not a UTI marker in hemodialysis-dependent ESRD patients. Urinary bacterial culture needs to be investigated for patients with positive pyuria findings. 
In our study, the mean postvoiding residual urine volume in UTI patients was $15.4 \mathrm{~mL}$, which was less than $50 \mathrm{~mL}$. May et $\mathrm{al}^{15}$ report that a postvoiding residual urine volume greater than $50 \mathrm{~mL}$ is a significant amount of urine and creates the potential for recurring UTI. Therefore, no relationship may exist between the postvoiding residual urine volume and the high proportion of UTI in hemodialysis-dependent ESRD patients. One of the causes of an increase in the postvoiding residual urine volume is the diabetic neurogenic bladder. ${ }^{16,17}$ Our study group contained 37 diabetic patients. The mean postvoiding residual urine volume among the 37 diabetic patients was $7.8 \mathrm{~mL}$. The postvoiding residual urine volume was small in hemodialysis-dependent ESRD patients. In our study, the mean daily urine output of the hemodialysis-dependent ESRD patients was $548 \mathrm{~mL}$ and $421.5 \mathrm{~mL}$ in normothermia and UTI, respectively. The mean daily urine output was understandably small in the hemodialysis-dependent ESRD patients. A speculation is that the increase in the postvoiding residual urine volume depends on a large quantity of daily urine output. Therefore, checking the postvoiding residual urine volume may not be needed routinely for hemodialysis-dependent ESRD patients who have a small amount of urine. However, one hemodialysis-dependent ESRD male patient was 72 years old and his postvoiding residual urine volume was $150 \mathrm{~mL}$. He had diabetes and pyuria without a sense of residual urine. Physicians may need to check the postvoiding residual urine volume at least once for hemodialysis-dependent ESRD patients who have pyuria or bacteriuria.

Urinary bacterial cultures were widely detected from gram-negative to gram-positive in the hemodialysis-dependent ESRD patients. Our results revealed $75 \%$ gram-negative bacteria and $25 \%$ gram-positive bacteria. Table 4 , which is based on published reports and our results, shows 51.5\% gram-negative bacteria and 48.5\% gram-positive bacteria. Bacterial cystitis in hemodialysis-dependent ESRD patients is equivalent to complicated UTI. In our study, bacteriuria was defined as $\geq 10^{4} \mathrm{CFU} / \mathrm{mL}$ in male patients and $\geq 10^{5} \mathrm{CFU} / \mathrm{mL}$ in female patients, based on the Guidelines on Urological Infections by the European Association of Urology. ${ }^{8}$ Manhal et $\mathrm{al}^{3}$ reported bacteriuria, defined as $\geq 10^{5} \mathrm{CFU} /$ $\mathrm{mL}$, in all patients. Haider et $\mathrm{al}^{4}$ and Nakai et $\mathrm{al}^{13}$ reported bacteriuria, defined as $\geq 10^{4} \mathrm{CFU} / \mathrm{mL}$, in all patients. In all of the aforementioned authors' reports, bacterial cultures contained $\geq 10^{4} \mathrm{CFU} / \mathrm{mL}$. Therefore, we speculated the causative bacteria of UTI in hemodialysis-dependent ESRD patients from the authors' reports and we aggregated their results.

Of note, $17.5 \%$ (7/40) of patients had ESBL-producing Escherichia coli, especially women, of whom 19.2\% (5/26) had ESBL-producing Escherichia coli. Wada et al ${ }^{18}$ reported that 6.5\% (55/847) ESBL-producing Escherichia coli were detected among non-ESRD women with acute uncomplicated cystitis in a Japanese nationwide survey. Song et al ${ }^{19}$ reported that $6.26 \%(24 / 383)$ of non-ESRD female outpatients with intractable recurrent cystitis had ESBL-producing Escherichia coli. The proportion of resistant bacteria in causative organisms of UTI was higher among hemodialysisdependent ESRD patients than among non-ESRD patients. This factor may be the main reason that ESRD patients usually visit hospitals and may be prescribed antibacterial drugs on many occasions. To prevent the outbreak of resistant

Table 4 All Published Reports of the Bacterial Cultures of Hemodialysis-Dependent ESRD Patients with Bacteriuria

\begin{tabular}{|c|c|c|c|c|c|}
\hline Author & Manhal et $\mathrm{al}^{3} \mathrm{n}$ & Haider et $\mathrm{al}^{4} \mathrm{n}$ & Nakai et al ${ }^{13} n$ & Present Study n & Total n (\%) \\
\hline Gram-negative & 13 & 12 & 13 & 30 & $68(5 \mid .5)$ \\
\hline Escherichia coli & 6 & 8 & 5 & 18 & $37(28.1)$ \\
\hline Klebsiella spp. & 5 & 3 & I & 5 & $14(10.7)$ \\
\hline Acinetobacter spp. & 1 & 0 & 0 & 2 & $3(2.2)$ \\
\hline Proteus spp. & 1 & I & 0 & 1 & $3(2.2)$ \\
\hline Pseudomonas aeruginosa & 0 & 0 & 1 & 2 & $3(2.2)$ \\
\hline Citrobacter spp. & 0 & 0 & 1 & 1 & $2(1.5)$ \\
\hline Enterobacter cloacae & 0 & 0 & 0 & 1 & $\mathrm{I}(0.8)$ \\
\hline Other & 0 & 0 & 5 & 0 & $5(3.8)$ \\
\hline Gram-positive & 2 & 14 & 38 & 10 & $64(48.5)$ \\
\hline Staphylococcus spp. & 1 & 7 & 22 & I & $31(23.5)$ \\
\hline Enterococcus spp. & 0 & 6 & 12 & 5 & $23(17.4)$ \\
\hline Streptococcus spp. & 1 & I & 3 & 4 & $9(6.8)$ \\
\hline Other & 0 & 0 & 1 & 0 & $\mathrm{I}(0.8)$ \\
\hline
\end{tabular}

Abbreviation: ESRD, end-stage renal disease. 
bacteria, antibacterial drugs should be prescribed for bacterial cystitis only when certain symptoms such as pollakisuria, urgency, sense of residual urine, and miction pain are aggravated.

For hemodialysis-dependent ESRD patients who have bacterial cystitis, which is equivalent to a complicated UTI, the choice of antibacterial drug for the treatment ${ }^{20}$ is to prescribe oral levofloxacin or ciprofloxacin as the first-choice therapy, ${ }^{20,21}$ and oral cefdinir or cefpodoxime proxetil as the second-choice therapy. ${ }^{20}$

The number of UTIs caused by multidrug-resistant microbial strains has significantly risen in recent years. ${ }^{2}$ ESBL bacteria are a beta-lactamase-producing strain. The emergence of strains expressing ESBL has been one of the most important developments in resistance and have become a global public health concern. ${ }^{2,21}$ Klebsiella, Escherichia, Enterobacter, Citrobacter, Serratia, Proteus, Morganella, and Providencia have antibiotic-resistance genes, which comprises the ESBLs. ${ }^{2,21}$ Carbapenem antibacterial drugs such as meropenem are prescribed as the first-choice therapy for UTIs caused by ESBL-producing strains, ${ }^{20,21}$ and cephamycins such as cefmetazole are prescribed for patients who have UTIs and who have acceptable vital signs. ${ }^{20} \mathrm{Jia}$ et al ${ }^{22}$ detected 308 ESBL-producing Escherichia coli among nonESRD patients. The susceptible rate of three hundred eight ESBL-producing Escherichia coli strains were 99.9\% and 97.9\% for imipenem and cefmetazole, respectively. Hamada et $\mathrm{al}^{23}$ reported that, among non-ESRD outpatients, cefmetazole was an efficacious treatment for UTI due to ESBL-producing Escherichia coli. Kuwana et al ${ }^{24}$ concluded that, for sepsis in UTI caused by ESBL-producing Escherichia coli among non-ESRD outpatients, de-escalation therapy from broad-spectrum antibiotics to cephamycins is a potential treatment when vital signs are stable. Our results showed that $100 \%$ of the seven ESBL-producing strains were susceptible to not only meropenem but also cefmetazole in hemodialysis-dependent ESRD patients. Cefmetazole, as well as meropenem, might be useful for UTI caused by ESBLproducing Escherichia coli, even in hemodialysis-dependent ESRD patients.

Behzadi $^{25}$ said that understanding the structures and mechanisms of fimbriae in uropathogenic Escherichia coli enables clinicians to prevent the occurrence of infections and/or to treat UTIs. FimH is bacterial adhesin that helps bacteria to bind to host cells, and is displayed on the bacterial surface as a component of the fimbrial organelle. ${ }^{25}$ Sarshar et $\mathrm{al}^{26}$ reported that mannose-based FimH antagonists, which are an antiadhesive therapeutic against uropathogens, are valuable and effective for treating UTIs in vitro and in vivo as an alternative to the antibiotic therapeutic strategy. We would like to investigate the usefulness of antiadhesive therapeutics for treating hemodialysis-dependent ESRD patients who have UTIs in the future.

Our study has several limitations. First, we may have included patients who had certain risk factors and comorbidities for UTIs such as neurological bladder or cystocele. However, diagnosing neurological bladder or cystocele is difficult without further invasive examinations. Second, in this study, we diagnosed patients who had both pyuria, based on urinalysis findings, and bacteriuria as a UTI, based on urinary bacterial culture findings. The patients did not have miction pain or fever. They may have been actually diagnosed as having asymptomatic cystitis, which is a type of UTI. However, previous investigators have used the term "urinary tract infection" for hemodialysis-dependent ESRD patients who did not any symptoms but had pyuria and bacteriuria. We matched previous papers as a matter of convenience. Third, we could not investigate the history of UTIs of the patients. The patients who had bacteriuria at the time of this study may develop pyelonephritis or urosepsis in the future. Thus, we would like to further investigate the history of UTIs for the patients in the long term.

\section{Conclusions}

The rate of UTI in dialysis-dependent ESRD patients was $32 \%$ and $20 \%$, in female patients and male patients, respectively. The proportion of resistant bacteria in causative organisms of UTI was high in dialysis-dependent ESRD patients. The urine output of hemodialysis-dependent ESRD patients decreased gradually; therefore, a urinary bacterial culture should be routinely checked while they can void some urine, even if they do not complain about bladder and urethral pain, especially for female patients. This finding is useful for the choice of antibacterial drug for treating UTIs in dialysis-dependent ESRD patients, and it will help the patient's life if they develop a UTI. 


\section{Abbreviations}

UTI, urinary tract infection; ESRD, end-stage renal disease; CFU, colony forming units; ESBL, extended spectrum betalactamase.

\section{Disclosure}

The authors have no conflicts of interest directly relevant to the content of this article.

\section{References}

1. Issakhaniam L. Behzadi. antimicrobial agents and urinary tract infections. Curr Pharm Des. 2019;25:1409-1423. doi:10.2174/ 1381612825999190619130216

2. Behzadi P, Garcia-Perdomo HA, Karpinski TM, Issakhanian L. Metallo-ß- lactamases: a review. Mol Biol Rep. 2020;47:6281-6294. doi:10.1007/ s11033-020-05651-9

3. Manhal FS, Mohammed AA, Ali KH. Urinary tract infection in hemodialysis patients with renal failure. J Fac Med Baghdad. 2012;54(1):38-41.

4. Haider JS, Osman EHA, Tahir KB. Frequency of urinary tract bacterial infection and their susceptibility patterns among hemodialysis patients in Zliten hospital. J Microbiol Exp. 2016;3(3):93-97.

5. Kong J, Davies M, Mount P. The importance of residual kidney function in haemodialysis patients. Nephrology. 2018;23:1078-1080. doi:10.1111/ nep. 13427

6. Pan Y, Hong YC, Shin HJ, et al. Malakoplakia and xanthogranulomatous pyelonephritis treated with nephrectomy A case report. Medicine. 2021;100(36):e27137. doi:10.1097/MD.0000000000027137

7. Midturi JK, Ranganath S. Prevention and treatment of multidrug-resistant organisms in end-stage renal disease. Adv Chronic Kidney Dis. 2019;26 (1):51-60. doi:10.1053/j.ackd.2018.09.003

8. Grabe M, Bartoletti R, Bjerklund Johansen TE, et al., editors. Guidelines on Urological Infections. The Netherlands: European Association of Urology; 2020.

9. Kuo IC, Lee JJ, Hwang DY, et al. Pyuria, urinary tract infection and renal outcome in patients with chronic kidney disease stage 3-5. Sci Rep. 2020;10(1):19460. doi:10.1038/s41598-020-76520-5

10. Gilbert DN. Urinary tract infections in patients with chronic renal insufficiency. Clin J Am Soc Nephrol. 2006;1(2):327-331. doi:10.2215/ CJN.01931105

11. Kwon YE, Oh DJ, Kim MJ, Choi HM. Prevalence and clinical characteristics of asymptomatic pyuria in chronic kidney disease. Ann Lab Med. 2020;40(3):238-244. doi:10.3343/alm.2020.40.3.238

12. Mortazavi M, Seyrafian S, Shahidi S, Abadpour Z, Shahbazi F. Pyuria as a screening test for detection of urinary tract infection in patients on long-term hemodialysis. Iran J Kidney Dis. 2011;5(1):50-52.

13. Nakai Y, Kagebayashi Y, Matsumoto Y, et al. Urinalysis and urinary bacterial culture in patients on maintenance hemodialysis. J Jpn Soc Dial Ther. 2015;48(8):471-475. Japanese. doi:10.4009/jsdt.48.471

14. Oikonomou KG, Alhaddad A. The diagnostic value of urinalysis in hemodialysis patients with fever, sepsis or suspected urinary tract infection. J Clin Diagn Res. 2016;10(10):11-13.

15. May M, Brookman-Amissah S, Hoschke B, Gilfrich C, Braun KP, Kendel F. Post-void residual urine as a predictor of urinary tract infection- is there a cutoff value in asymptomatic men? $J$ Urol. 2009;181(6):2540-2544. doi:10.1016/j.juro.2009.01.103

16. Lee WC, Wu HP, Tai TY, Yu HJ, Chiang PH. Investigation of urodynamic characteristics and bladder sensory function in the early stages of diabetic bladder dysfunction in women with type 2 diabetes. $J$ Urol. 2009;181(1):198-203. doi:10.1016/j.juro.2008.09.021

17. Ichihara K, Takahashi S, Masumori N. Pathophysiology and management of urinary tract infection in patients with diabetic bladder dysfunction. Jpn J Chemother. 2016;64:730-734. Japanese.

18. Wada K, Yokoyama T, Uno S, et al. Nationwide surveillance of bacterial pathogens isolated from patients with acute uncomplicated cystitis in 2018: conducted by the Japanese Research Group for urinary tract infections (JRGU). J Infect Chemother. 2021;27(8):1169-1180. doi:10.1016/j. jiac.2021.03.012

19. Song S, Kim C, Lim D. Clinical efficacy of Ertapenem for recurrent cystitis caused by multidrug-resistant extended-spectrum $\beta$-lactamaseproducing Escherichia coli in female outpatients. Korean J Urol. 2014;55(4):270-275. doi:10.4111/kju.2014.55.4.270

20. Aoki N, Arakawa S, Onai K, et al., editors. JAID/ISC Guide to Clinical Management of Infectious Diseases 2019. Tokyo: Life science publishers; 2019. Japanese.

21. Behzadi P, Urban E, Matuz M, Gajdacs M. The role of gram-negative bacteria in urinary tract infections: current concepts and therapeutic options. Adv Exp Med Biol. 2021;15:35-69.

22. Jia P, Zhn Y, Li X, et al. High prevalence of extended-spectrum beta-lactamases in Escherichia coli strains collected from strictly defined community-acquired urinary tract infections in adults in China: a multicenter prospective clinical microbiological and molecular study. Front Microbiol. 2021;12:663033. doi:10.3389/fmicb.2021.663033

23. Hamada Y, Matsumura Y, Nagashima M, Akazawa T, Doi Y, Hayakawa K. Retrospective evaluation of appropriate dosing cefmetazole for invasive urinary tract infection due to extended-spectrum $\beta$-Lactamases in Escherichia coli. J Infect Chemother. 2021;27(11):1602-1606. doi:10.1016/j. jiac.2021.07.009

24. Kuwana T, Yamaguchi J, Kinoshita K, Hori A, Ihara S, Taniguchi T. Successful de-escalation antibiotic therapy using cephamycins for sepsis caused by extended-spectrum beta-lactamase-producing Enterobacteriaceae bacteremia: a sequential 25-case series. Open Med. 2020;15 (1):782-786. doi:10.1515/med-2020-0103

25. Behzadi P. Classical chaperone-usher (CU) adhesive fimbriome: uropathogenic Escherichia coli (UPEC) and urinary tract infections (UTIs). Folia Microbiol. 2020;65:45-65. doi:10.1007/s12223-019-00719-x

26. Sarshar M, Behzadi P, Ambrosi C, Zagaglia C, Palamara AT, Scribano D. FimH and anti-adhesive therapeutics: a disarming strategy against Uropathogens. Antibiotics. 2020;9(7):397. doi:10.3390/antibiotics9070397 


\section{Publish your work in this journal}

Research and Reports in Urology is an international, peer-reviewed, open access journal publishing original research, reports, editorials, reviews and commentaries on all aspects of adult and pediatric urology in the clinic and laboratory including the following topics: Pathology, pathophysiology of urological disease; Investigation and treatment of urological disease; Pharmacology of drugs used for the treatment of urological disease. The manuscript management system is completely online and includes a very quick and fair peer-review system, which is all easy to use. Visit http://www.dovepress.com/testimonials.php to read real quotes from published authors.

Submit your manuscript here: https://www.dovepress.com/research-and-reports-in-urology-journal 